\title{
FLUXO DIFUSIVO DE ZINCO EM AMOSTRAS DE SOLO INFLUENCIADO POR TEXTURA, ÍON ACOMPANHANTE E PH DO SOLO(1)
}

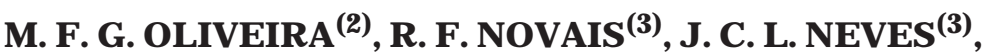 \\ V.M.C. ALVES ${ }^{(4)} \&$ C. A. VASCONCELLOS $(4)$
}

\begin{abstract}
RESUMO
O fluxo difusi vo (difusão) é a forma mais importante de transporte de zinco no solo, dada sua baixa concentração na solução. E ste trabal ho teve como objetivo avaliar o fluxo difusivo de $\mathrm{Zn}$ em amostras de um solo de textura argilosa (Latossolo Vermel ho-E scuro - LE), outro de textura média (Podzólico VermelhoAmarelo - PV) e um terceiro de textura arenosa (L atossolo Vermel ho-Amarelo LV), submetidos a níveis de pH: pH natural $(4,38)$ e 5,40 , para o LE; natural $(4,87)$ e 6,00, para o PV, e somente $\mathrm{pH}$ natural $(4,64)$, para o $\mathrm{LV}$, e a três fontes de $\mathrm{Zn}$ $\left(\mathrm{ZnCl}_{2}, \mathrm{ZnEDTA}\right.$ e $\left.\mathrm{ZnSO}_{4}\right)$ nas doses de 0, 20 e $40 \mathrm{mg} \mathrm{dm}^{-3}$ de $\mathrm{Zn}$. As unidades experimentais constituíram-se de $400 \mathrm{~cm}^{3}$ de amostras de solo, colocadas em câmaras feitas de tubos de PVC, com $10 \mathrm{~cm}$ de diâmetro $5 \mathrm{~cm}$ de altura, contendo, cada câmara, uma lâmina de resina trocadora de cátions ácido forte (modelo CR61CZR IONI CS, Inc) como dreno de Zn, nas dimensões de 2,0 x 5,0 cm, colocada à profundidade de $2,5 \mathrm{~cm}$ no meio da câmara. As amostras, umedecidas até a capacidade de campo, foram incubadas por um período de 15 dias à temperatura de $\mathbf{2 4} \pm 4^{\circ} \mathrm{C}$. Após esse período, as lâminas foram retiradas, realizando-se a extração do $\mathrm{Zn}$ adsorvido às lâminas de resina. $\mathbf{O}$ pH do solo demonstrou ser fator de grande importância no controle do fluxo difusivo do $\mathrm{Zn}$, acarretandoIhe grande diminuição quando da elevação de pH. De modo geral, o fluxo difusivo de $\mathrm{Zn}$ foi menor com o aumento do teor de argila do solo. $\mathbf{O}$ fluxo difusivo de $\mathrm{Zn}$ foi maior, nos três solos, quando a fonte utilizada foi $\mathrm{ZnCl}_{2}$.
\end{abstract}

Termos de indexação: difusão, fontes de zinco, acidez, resina.

(1) Recebido para publicação em setembro de 1998 e aprovado em fevereiro de 1999.

(2) Engenheiro-Agrônomo, Doutorando em Solos e Nutrição de Plantas, Departamento de Ciência do Solo, Universidade Federal de Lavras (UFLA). CEP 37200-000 Lavras (MG).

(3) Professor do Departamento de Solos, Universidade Federal de Viçosa. CEP 36571-000 Viçosa (MG).

(4) Pesquisador da Embrapa Milho e Sorgo. Rodovia MG 424 Km 65, Caixa Postal 151, CEP 35701-970 Sete Lagoas (MG). 


\title{
SUMMARY: DIFFUSIVE FLUX OF ZINCIN SOIL SAMPLES AS A FUNCTION OF TEXTURE, COUNTER ION AND SOIL PH
}

\begin{abstract}
The diffusi ve flux (diffusion) is the main form of zinc transport in soil, due to its low concentration in solution. This work aimed to evaluate the diffusion of $\mathrm{Zn}$ in samples of thresoil types: a loamy textureDark-Red Latosol (LE), a medium textureRed-Yellow Podzolic (PV), and a sandy texture Red-Y ellow Latosol (LV). The diffusion was assessed in samples with thenatural $\mathrm{pH}$ for thethreesoil samples and at pH 5.40 for the LE and 6.00 for thePV. Threesources of $\mathrm{Zn}\left(\mathrm{ZnCl}_{2}, \mathrm{ZnEDTA}\right.$ and $\left.\mathrm{ZnSO}_{4}\right)$ at therates of 0,20 and $40 \mathrm{mg} \mathrm{dm}^{-3}$ of $\mathrm{Zn}$ weretested. Theexperimental units wereconstituted of $400 \mathrm{~cm}^{3}$ of soil pl aced in PVC rings, $10 \mathrm{~cm}$ of diameter and $5 \mathrm{~cm}$ high. At the depth of $2.5 \mathrm{~cm}$, a slide of a strong acid exchange cation resin (CR61CZR IONICS, I NC.) as a sink for Zn, with the dimensions of $2.0 \times 5.0 \mathrm{~cm}$ was set. The soil samples were moistened to the field capacity and incubated for 15 days. Then, theresin slides were removed and submitted to the extraction of $\mathrm{Zn}$. Soil pH was an important factor control ling Zn diffusion, which decreased with $\mathrm{pH}$ increase In general, $\mathrm{Zn}$ diffusive flux was lower with the increase of the soil clay content. The diffusive flux of $\mathrm{Zn}$ was greater in the threesoils, when thesource was $\mathrm{ZnCl}_{2}$.
\end{abstract}

Index terms: diffusion, sources of zinc, acidity, resin.

\section{INTRODUÇÃO}

Sintomas dedeficiência de zinco em plantas estão relacionados, principalmente, com a baixa disponibilidade desse nutriente nos sol os, a qual, por sua vez, está relacionada com o tipo de reações do el emento aos componentes do sol o e com sua forma detransporte. $\mathrm{O}$ entendimento de como essas reações acontecem é essencial à correção de deficiências desse el emento nos cultivos.

O fluxo difusivo é a forma de maior importância para otransporte deZn, dada sua baixa concentração na solução do solo (Oliver \& Barber, 1966; Sharma \& Deb, 1984; Marschner, 1993). Em dois solos com 10,3 e $14 \%$ de argila e pH 8,4 e 8,5, Sharma \& Deb (1987) registraram uma contribuição de transporte de $\mathrm{Zn}$ para trigo de 9,8 e 18,5\% pel o fluxo de massa e de 81,5 e $90,2 \%$ pela difusão.

Os processos de adsorção de Zn a constituintes do solo são o principal mecanismo de controle da dinâmica do Zn no solo. Para Cunha (1989), os mecanismos envolvidos na adsorção podem estar relacionados com a adsorção el etrostática ou específica. De modo geral, no entanto, a adsorção específica é o principal mecanismo de retenção de Zn no solo. Para Ellis \& Knezek (1972), a adsorção específica dá-se pela ligação do $\mathrm{Zn}$ com grupos OH octaedral em argilas silicatadas, em faces quebradas, em aberturas e em defeitos na estrutura cristalina. Essa adsorção específica pode ser esquematicamente representada por: $\left(\begin{array}{cl}\mathrm{Al}-\mathrm{O}-\mathrm{Zn} \\ \mathrm{H}^{+}\end{array}\right)$

A adsor ção de Zn por argilas silicatadas tem sido registrada na literatura. A presença de óxidos de Fe e de Al influencia essa adsorção (Ferreira \& Cruz, 1991). A remoção de óxidos de $\mathrm{Fe}$ eleva, com mais freqüência que diminui, a capacidade de adsorção
deZn pelas argilas silicatadas. Em minerais com Al em arranjos octaedrais, o $\mathrm{Zn}$ pode ser fixado em espaços não ocupados pelo alumínio na estrutura. Esse tipo de fixação é acompanhado por um decréscimo na capacidade de adsorção de cátions e por um aumento na capacidade de adsorção de ânions pelo mineral (Elgabaly, 1950).

Maior deficiência de $\mathrm{Zn}$ em plantas ocorre em sol os com valores de pH acima de 6,0, sendo os solos calcários mais propensos a esse problema. A pH elevado, o $\mathrm{Zn}$ forma compostos insolúveis como $\mathrm{Zn}(\mathrm{OH})_{2} \mathrm{eZnCO}_{3}$ (Lindsay, 1991). Deve-se observar, no entanto, que nem todos os sol os com pH alcalino apresentam problemas de deficiência de $\mathrm{Zn}$ decorrentes do mecanismo de quelatação do Zn que ocorre naturalmente com substâncias orgânicas, compensando, dessa forma, sua baixa solubilidade nessas condições.

Considerando as reações de hidrólise de compostos de Zn no solo, conclui-se que, para cada aumento de uma unidade de $\mathrm{pH}$, a solubilidade das formas de $\mathrm{Zn}$ no solo cai, aproximadamente, 100 vezes (Lindsay, 1979). Segundo Moraghan \& Mascagni J r. (1991), na faixa de $\mathrm{pH}$ de 5,5 a 7,0, a concentração de equilíbrio do Zn diminui de 30 a 45 vezes com o aumento de cada unidade de $\mathrm{pH}$ do solo. A difusão de $\mathrm{Zn}$ em solos calcários pode, portanto, ser drasticamente menor que em solos ácidos, e a calagem de sol os ácidos pode levar a um decréscimo no coeficiente de difusão semel hante ao encontrado em solos calcários.

Cátions metálicos podem formar pares iônicos e quel atos com ânions inorgânicos ou orgâni cos (Tam, 1992). Dessa associação, podem resultar compostos positiva ou negativamente carregados, ou mesmo sem carga. Os complexos resultantes podem ser 
fracamente adsorvidos à superfície dos minerais ou, ainda, adsorvidos mais fortemente que o elemento em sua forma iônica. Para Lindsay (1979), quelatos orgânicos, comoaqueles com citratoe oxalato, podem desempenhar importante papel na mobilidade dos metais.

É sabido que íons acompanhantes influem na seletividade de troca de uma superfície coloidal (Tisdale et al., 1985). Kalbasi et al. (1978), citados por Cunha (1989), constataram que a solubilidade do $\mathrm{Zn}$ era dependente dos ânions presentes na sol ução do solo, tendo sido observada a seqüência de solubilidade: $\mathrm{ZnS}<\mathrm{ZnSO}_{4}<\mathrm{ZnEDTA}$. O EDTA forma com o Zn complexos al tamente estáveis, sendo mai or a estabilidade com o aumento do pH. Em seu trabalho, Kumari et al. (1988), utilizando um solo siltoso e um argiloso, ambos de origem aluvial e alcalinos, encontraram maior difusão no sol o siltoso. Observou-se a seguinte ordem de grandeza de difusão, para as diferentes fontes de $Z n$ utilizadas: $\mathrm{ZnCl}_{2}>\mathrm{ZnCO}_{3}>\mathrm{ZnSO}_{4}>\mathrm{Zn}\left(\mathrm{NO}_{3}\right)_{2}>\mathrm{Zn}(\mathrm{OAC})_{2}$.

Este trabalho teve como objetivo verificar a influência de diferentes íons acompanhantes do Zn, da variação do $\mathrm{pH}$ e da textura do sol o sobre o fluxo difusivo deste el emento em amostras de solos.

\section{MATE RIAL E MÉTODOS}

F oram utilizadas amostras de três sol os $(0-20 \mathrm{~cm}$ de profundidade): um Latossolo Vermelho-Escuro (LE), de Sete Lagoas, um Podzólico VermelhoAmarelo (PV), de Ponte Nova, e um Latossolo Vermelho-Amarelo (LV), de Três Marias, MG. Os sol os foram secos ao ar, destorroados, passados em peneira de $2 \mathrm{~mm}$ de mal ha ehomogeneizados. Dessas amostras, retiraram-se subamostras que foram submetidas a análises quími cas efísicas (Quadro 1).

Nos extratos, os teores de ferro foram dosados por espectrofotometria de absorção atômica; os de alumínio, por espectrofotometria de absorção molecular, pelo método do aluminon (Alvarez V., 1985), e os de fósforo, por espectrofotometria de absorção molecular do complexo fosfomolíbdico, reduzido pelo ácido ascórbico (Braga \& Defelipo, 1974). Cálcio e magnésio trocáveis extraídos foram determinados por absorção atômica e alumínio trocável por titulometria (EMBRAPA, 1979).

Amostras do $\mathrm{LE}$ ePV receberam $\mathrm{CaCO}_{3}$ p.a., com vistas em elevar em uma unidade seus valores iniciais de pH (4,38 e 4,87, respectivamente), em dose estimada por curva de incubação. Depois de 30 dias de incubação dos sol os com o corretivo, foram obti dos os valores de pH 5,40, para o LE, e 6,00, para oPV. $\mathrm{O}$ LV foi incluído no estudo apenas em seu $\mathrm{pH}$ natural $(4,64)$ como comparação com os demais sol os quanto ao efeito de textura sobre ofluxo difusivo de Zn nos solos.
Três fontes de $\mathrm{Zn}\left(\mathrm{ZnCl}_{2}\right.$, ZnEDTA e $\left.\mathrm{ZnSO}_{4}\right)$ foram aplicadas aos solos nas doses de 0, 20 e $40 \mathrm{mg} \mathrm{dm}^{-3}$. Essas fontes de Zn foram homogeneizadas com as amostras dos solos e deixadas em incubação com a umidade dos solos el evada à capacidade de campo (Quadro 1), por 30 dias.

Após a incubação, as amostras foram secas, passadas em peneira de $1 \mathrm{~mm}$, sendo, então, colocadas em câmaras feitas de tubos de PVC com $10 \mathrm{~cm}$ de diâmetro e cinco cm de altura, com uma chapa de isopor no fundo. Para a montagem das câmaras, colocou-se metade do solo $\left(200 \mathrm{~cm}^{3}\right.$, aproximadamente), uma lâmina de resina trocadora de cátions, ácido forte (CR61CZR IONICS, Inc.), nas dimensões de 2,0 x 5,0 cm (com capacidade máxima de adsorção de 0,125 mg cm-2 de Zn, considerandose as duas faces da lâmina) e sobre esta o restante do sol o. As amostras desol oforam, então, umedecidas até a capacidade de campo e envolvidas em filme plástico para evitar perda de umidade durante o período de contato da lâmina de resina com o solo. Após um período de 15 dias, a uma temperatura média de $24 \pm 4^{\circ} \mathrm{C}$, as lâminas foram retiradas, lavadas com água destilada e colocadas em solução extratora, segundo Raij \& Quaggio (1983), realizando-se a extração do $Z n$ adsorvido na resina e sua determinação por espectrofotometria de absorção atômica.

O experimento constou de um fatorial $[(1 \times 2)+$ $(1 \times 2)+(1 \times 1)] \times[1+(3 \times 3)]$, respectivamente: [LE $x$ calagem (sem e com corretivo), PV x calagem (sem e com corretivo) e LV sem corretivo] [dose zero de Zn nos três solos e as três fontes nas dose 0,20 e $40 \mathrm{mg} \mathrm{kg}^{-1}$ de Zn]. Foram feitas três repetições, em um delineamento em blocos casualizados.

\section{RESULTADOSE DISCUSÃO}

A calagem foi o fator de maior importância no controle do fluxo difusivo (Quadros 2 e 3 ) em comparação aos fatores solo e íon acompanhante (fontes de Zn). Nas amostras com calagem, o fluxo difusivo de Zn apresentou diminuição acentuada em relação às amostras sem calagem. Resultados semel hantes foram registrados por Machado \& Pavan (1987). Esses autores observaram di minuição na solubilidade de $\mathrm{Zn}$ e aumento na capacidade máxima de adsorção e na energia de ligação do Zn com o solo em resposta à aplicação de calcário e conseqüente el evação do $\mathrm{pH}$ do solo. Relataram, ainda, que o aumento da adsorção do Zn com a el evação do pH do solo pode estar relacionado com uma maior afinidade dos sítios de troca pela forma hidrolisada com menor valência $\left(\mathrm{ZnOH}^{+}\right)$.

Maguire et al. (1981) registraram que o aumento do poder deadsorção de Zn pel o solo, com o aumento do $\mathrm{pH}$, tem efeito mais marcante que a precipitação de Zn em formas insolúveis. 
Quadro 1. Características químicas e físicas das amostras dos solos

\begin{tabular}{|c|c|c|c|}
\hline \multirow{2}{*}{ Característica } & \multicolumn{3}{|c|}{ Solo } \\
\hline & LE & PV & LV \\
\hline $\mathrm{pH}$ em $\mathrm{H}_{2} \mathrm{O}(1: 2,5)$ & 4,38 & 4,87 & 4,64 \\
\hline $\mathrm{pH}$ em KCl $1 \mathrm{~mol} \mathrm{~L}^{-1}(1: 2,5)$ & 3,83 & 4,92 & 4,92 \\
\hline P Mehlich-1 $\left(\mathrm{mg} \mathrm{dm}^{-3}\right)^{(1)}$ & 1,3 & 1,3 & 0,7 \\
\hline $\mathrm{P}$ remanescente $\left(\mathrm{mg} \mathrm{L}^{-1}\right)^{(2)}$ & 3,2 & 31,6 & 38,1 \\
\hline $\mathrm{Zn}-\mathrm{DTPA}\left(\mathrm{mg} \mathrm{dm}^{-3}\right)^{(3)}$ & 0,227 & 0,378 & 0,061 \\
\hline $\mathrm{Zn}-\mathrm{HCl}\left(\mathrm{mg} \mathrm{dm}^{-3}\right)^{(4)}$ & 0,361 & 0,566 & 0,0 \\
\hline Zn-Mehlich-1 (mg dm $\left.{ }^{-3}\right)^{(1)}$ & 0,496 & 0,642 & 0,072 \\
\hline Zn-Mehlich-3 $\left(\mathrm{mg} \mathrm{dm}^{-3}\right)^{(5)}$ & 0,276 & 1,182 & 0,039 \\
\hline $\mathrm{Al}^{3+}\left(\mathrm{mmol}_{\mathrm{c}} \mathrm{dm}^{-3}\right)^{(6)}$ & 17,4 & 4,0 & 7,5 \\
\hline $\mathrm{Ca}^{2+}\left(\mathrm{mmol}_{\mathrm{c}} \mathrm{dm}^{-3}\right)^{(6)}$ & 9,4 & 5,3 & 0,0 \\
\hline $\mathrm{Mg}^{2+}\left(\mathrm{mmol} \mathrm{dm}^{-3}\right)^{(6)}$ & 1,5 & 1,3 & 0,4 \\
\hline $\mathrm{H}+\mathrm{Al}\left(\mathrm{mmol}^{\left.\mathrm{dm}^{-3}\right)^{(7)}}\right.$ & 128,4 & 39,0 & 31,7 \\
\hline $\mathrm{S}\left(\mathrm{mmol}_{\mathrm{c}} \mathrm{dm}^{-3}\right)$ & 11,7 & 7,6 & 0,9 \\
\hline CTC efetiva $\left(\mathrm{mmol}_{\mathrm{c}} \mathrm{dm}^{-3}\right)$ & 29,1 & 11,6 & 8,4 \\
\hline CTC total $\left(\mathrm{mmol}_{\mathrm{c}} \mathrm{dm}^{-3}\right)$ & 157,5 & 46,6 & 40,1 \\
\hline $\mathrm{V}(\%)$ & 7,4 & 16,8 & 2,2 \\
\hline m (\%) & 59,8 & 34,6 & 89,3 \\
\hline C.O. $\left(\mathrm{g} \mathrm{kg}^{-1}\right)^{(8)}$ & 21,3 & 6,5 & 5,3 \\
\hline Areia grossa $\left(\mathrm{g} \mathrm{kg}^{-1}\right)^{(9)}$ & 80,0 & 500,0 & 500,0 \\
\hline Areia fina $\left(\mathrm{g} \mathrm{kg}^{-1}\right)^{(9)^{\prime}}$ & 70,0 & 220,0 & 360,0 \\
\hline Silte $\left(\mathrm{g} \mathrm{kg}^{-1}\right)^{(9)}$ & 130,0 & 60,0 & 20,0 \\
\hline Argila $\left(\mathrm{g} \mathrm{kg}^{-1}\right)^{(9)}$ & 720,0 & 220,0 & 120,0 \\
\hline Capacidade de campo $\left(\mathrm{kg} \mathrm{kg}^{-1}\right)^{(10)}$ & 0,37 & 0,18 & 0,15 \\
\hline Densidade aparente $\left(\mathrm{g} \mathrm{cm}^{-3}\right)^{(2)}$ & 0,96 & 1,22 & 1,39 \\
\hline
\end{tabular}

(1) Defelipo \& Ribeiro (1981). ${ }^{\text {(2) }}$ Alvarez V. (1982). (3) Liang \& Karamanos (1993). (4) Wu et al. (1991). ${ }^{\text {(5) }}$ Mehlich (1984). ${ }^{(6)}$ Extrator $\mathrm{KCl} 1 \mathrm{~mol} \mathrm{~L}^{-1}$ (Defelipo \& Ribeiro, 1981). ${ }^{(7)}$ Extrator: CaOAc 0,5 mol L-1, pH 7,0 (Defelipo \& Ribeiro, 1981). ${ }^{(8)}$ Método Walkley-Black (Defelipo \& Ribeiro, 1981). ${ }^{(9)}$ Método da Pipeta (EMBRAPA, 1979). ${ }^{(10)}$ Método da Coluna (Fernandes \& Sykes, 1968).

Cavallaro \& McBride (1984), trabalhando com um sol o com $10 \%$ de argila, observaram que, a partir de $\mathrm{pH}$ 5,0, a adsorção de $\mathrm{Zn}$ aumentava rapidamentee que, na faixa de $\mathrm{pH}$ de alta adsorção de Zn, entre 5,0 e 7,0, cerca de $95 \%$ do el emento adsorvido não pôde ser recuperado por meio de extrações sucessivas com solução de $\mathrm{CaCl}_{2}$ 0,05 mol L-1.

O menor efeito da el evação de $\mathrm{pH}$ sobre o fluxo difusivo de $\mathrm{Zn}$ foi de diminuí-lo praticamente cinco vezes para a dose de $10 \mathrm{mg} \mathrm{dm}^{-3}$, para a fonte $\mathrm{ZnSO}_{4}$, no PV, e o maior efeito foi de diminuí-lo 26 vezes para a dose de $40 \mathrm{mg} \mathrm{dm}^{-3}$, no LE, para a fonte $\mathrm{ZnCl}_{2}$ (Quadro 2). A diminuição do fluxo difusivo observado foi menor que os valores registrados por Moraghan \& Mascagni (1991), que indicaram diminuição do fluxo difusivo de Zn em 30 a 45 vezes para o aumento de cada unidade de $\mathrm{pH}$ do solo. Bear (1967) verificou maior adsorção de $\mathrm{Cl}^{-}$ por um sol o caul inítico para menores val ores de $\mathrm{pH}$; a adsorção de $\mathrm{SO}_{4}{ }^{2-}$ pel o mesmo solo foi, no mínimo, duas vezes e, no máximo, sete vezes maior que a do $\mathrm{Cl}^{-}$. Segundo Lindsay (1979), o $\mathrm{ZnCl}_{2}$ é altamente solúvel, o que explica a maior movimentação do Zn nesta forma.

Nas amostras sem aplicação de cal cário, o fluxo difusivo de $\mathrm{Zn}$ para as fontes $\mathrm{ZnCl}_{2} \mathrm{eZnSO}_{4}$ obedeceu à seguinte ordem de grandeza entre solos: LV (arenoso) > PV (textura média) > LE (argiloso)
Quadro 2. Fluxo difusi vo de zinco como variável de solo, pH, dose e íon acompanhante (fonte) após 15 dias de contato da lâmina de resina e o solo

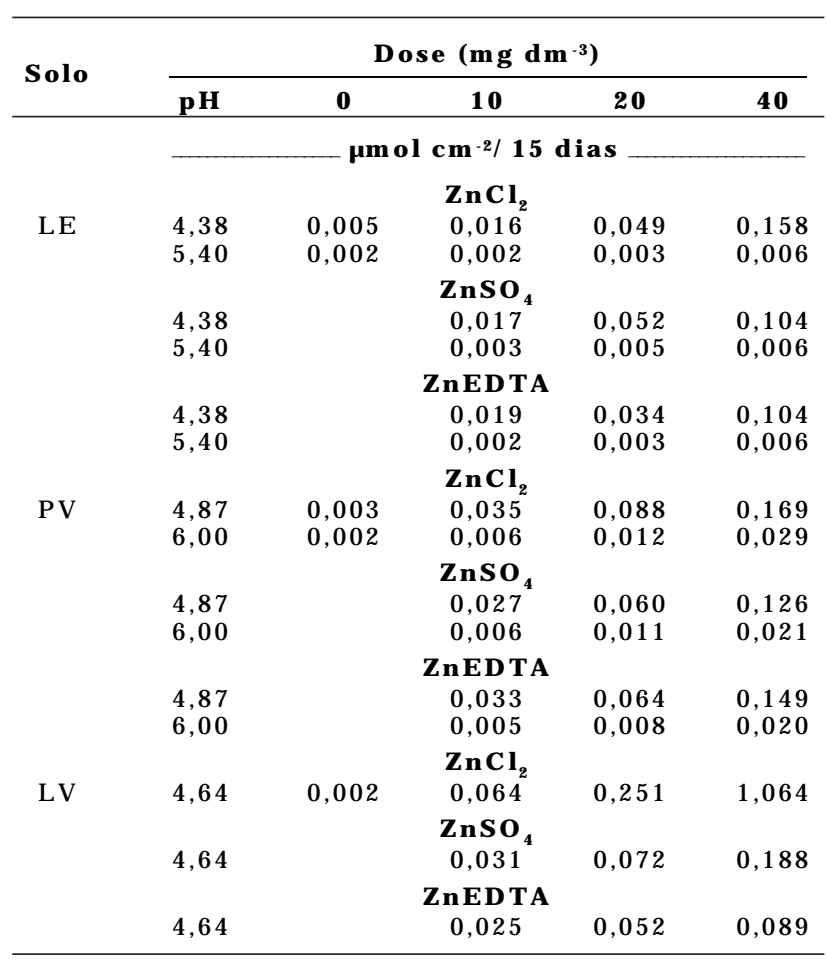


Quadro 3. Análise de variância do fluxo difusivo para os solos LE e PV, em presença e ausência de corretivo, e $L V$, sem corretivo: contraste entre fontes de zi $\mathrm{Co}\left(\mathrm{ZnCl}_{2}, \mathrm{ZnEDTA} \mathrm{e} \mathrm{ZnSO}_{4}\right)$ em cada solo e contrastes adicionais para solos dentro de fontes

\begin{tabular}{|c|c|c|c|c|c|c|c|}
\hline \multirow{2}{*}{ Fonte de variação } & \multirow{2}{*}{ G.L. } & \multicolumn{6}{|c|}{ Quadrado médio } \\
\hline & & $d / L E$ & $d / P V$ & $d / L V$ & $\mathrm{~d} / \mathrm{ZnCl}_{2}$ & d/ZnEDTA & $\mathrm{d} / \mathrm{ZnSO}_{4}$ \\
\hline & & \multicolumn{6}{|c|}{ Solo sem corretivo } \\
\hline Bloco & 2 & & $0,000676^{n s}$ & & & & \\
\hline Solo & 2 & & $0,142706^{* *}$ & & & & \\
\hline Dose vs Fonte & 1 & $0,0063^{*} *$ & $0,004246 * *$ & $1,801965 * *$ & & & \\
\hline $\mathrm{Cl}^{-}$vs (EDTA $\left.+\mathrm{SO}_{4}{ }^{2-}\right)$ & 1 & $0,007^{* *}$ & $0,00781^{* *}$ & $2,65^{* *}$ & & & \\
\hline EDTA vs $\mathrm{SO}_{4}{ }^{2-}$ & 1 & $0,000384^{\mathrm{ns}}$ & $0,0016^{\circ}$ & $0,0234 * *$ & & & \\
\hline Dose $\mathrm{d} / \mathrm{Cl}^{-}$ & 2 & $0,016432 * *$ & $0,013617 * *$ & $0,848229 * *$ & & & \\
\hline Dose d/EDTA & 2 & $0,00621^{* *}$ & $0,022006 * *$ & $0,003095 * *$ & & & \\
\hline Dose $\mathrm{d} / \mathrm{SO}_{4}^{2-}$ & 2 & $0,005699 * *$ & $0,007542 * *$ & $0,019892 * *$ & & & \\
\hline LV vs $\mathrm{PV}^{(1)}$ & 1 & & & & $1,76258 * *$ & $0,0103^{* *}$ & $0,00844 * *$ \\
\hline$P V$ vs $L E^{(1)}$ & 1 & & & & $0,0075615^{* *}$ & $0,0124 * *$ & $0,002646^{\circ}$ \\
\hline Erro & 58 & & 0,000695 & & & & \\
\hline \multirow[t]{2}{*}{ C.V. (\%) } & 25,1 & & & & & & \\
\hline & & \multicolumn{6}{|c|}{ Solo com corretivo } \\
\hline Bloco & 2 & & $0,000008^{n s}$ & & & & \\
\hline Solo & 1 & & $0,000877 * *$ & & & & \\
\hline Dose vs Fonte & 1 & $0,000001^{\mathrm{ns}}$ & $0,000157^{* *}$ & & & & \\
\hline $\mathrm{Cl}^{-}$vs $\left(\mathrm{EDTA}+\mathrm{SO}_{4}{ }^{2-}\right)$ & 1 & $0,000005^{\mathrm{ns}}$ & $0,000265^{* *}$ & & & & \\
\hline EDTA vs $\mathrm{SO}_{4}^{2-}$ & 1 & $0,000014^{\mathrm{ns}}$ & $0,000038^{* *}$ & & & & \\
\hline Dose $\mathrm{d} / \mathrm{Cl}^{-}$ & 2 & $0,000011 *$ & $0,000408 * *$ & & & & \\
\hline Dose d/EDTA & 2 & $0,00001^{*}$ & $0,00017^{* *}$ & & & & \\
\hline Dose $\mathrm{d} / \mathrm{SO}_{4}{ }^{2-}$ & 2 & $0,000008^{\mathrm{ns}}$ & $0,000189 * *$ & & & & \\
\hline PV vs LE ${ }^{(1)}$ & 1 & & & & $0,002^{* *}$ & $0,001^{* *}$ & $0,001 * *$ \\
\hline Erro & 38 & & 0,000003 & & & & \\
\hline C.V. (\%) & 22,6 & & & & & & \\
\hline
\end{tabular}

(1) Contrastes adicionais. ${ }^{\text {ns }},{ }^{\circ},{ }^{*}$ ** não-significativo, significativo a 10,5 e $1 \%$, respectivamente.

(Quadros 2 e3). Esses resultados são semelhantes a outros encontrados na literatura (Clarke\& Graham, 1968; Singh et al., 1980 eSharma \& Deb, 1990). Sol os mais argilosos apresentam maior número de sítios de troca, acarretando mai or adsorção de Zn e, como conseqüência, menor fluxo difusivo que os arenosos.

Não foi encontrada na literatura justificativa para a ordem decrescente de fluxo difusivo para a fonte de ZnEDTA (PV > LV ${ }^{15}$ ) LE). No entanto, segundo Lindsay (1972), a maior estabilidade de ZnEDTA ocorreu a pH 6,5, situação em que aproximadamente $70 \%$ do EDTA ligante estaria quelatado com Zn. Abaixo deste val or de pH, o Zn²+ ligado a EDTA pôde ser deslocado (substituído) por $\mathrm{Fe}^{3+} \mathrm{e}$, em altos valores, por $\mathrm{Ca}^{2+}$.

(5) Contraste não previsto anteriormente à análise estatística; significativo a $1 \%$ pelo teste $\mathrm{F}$.
A ordem de grandeza do fluxo difusivo de Zn em função do íon acompanhante (Quadros 2 e 3) foi para o solo $\mathrm{LE}: \mathrm{Cl}^{-}>\mathrm{EDTA}=\mathrm{SO}_{4}{ }^{2-}$; para o solo $\mathrm{PV}: \mathrm{Cl}^{-}$ $>$ EDTA $>\mathrm{SO}_{4}{ }^{2-}$ e para osolo LV: $\mathrm{Cl}^{-}>\mathrm{SO}_{4}{ }^{2-}>$ EDTA. $\mathrm{O}$ fluxo difusivo de $\mathrm{Zn}$ para a fonte $\mathrm{ZnCl}_{2}$ foi maior em todos os solos, como também encontrado por Kumari et al. (1988). Tal fato pareceser conseqüência da baixa capacidade de adsorção de $\mathrm{Cl}^{-}$pel os solos, em geral (Elgabaly, 1950), e da alta solubilidade do $\mathrm{ZnCl}_{2}$ (Lindsay, 1979).

Para o L atossolo Vermelho-Escuro, o mais argiloso, ofluxo difusivo de Zn para as fontes ZnEDTA eZnSO nãodiferiram entresi (Quadros 2e3). Comodiscutido, a menor movimentação de ZnEDTA neste solo em relação a $\mathrm{ZnCl}_{2}$ deveu-se à possível substituição do $\mathrm{Zn}^{2+}$ no quelato por $\mathrm{Fe}^{3+}$. Tal substituição ocorre em sol os com pH mais ácido (Lindsay, 1972). No solo de textura média (PV), observou-separa ZnEDTA maior mobilidade que para $\mathrm{ZnSO}_{4}$ como conseqüência da menor disponibilidade de $\mathrm{Fe}^{3}+$ neste solo, permitindo 
Quadro 4. Equações de regressão ajustadas para valores do fluxo difusivo ( $\mu \mathrm{mol} \mathrm{cm}^{-2} / 15$ dias) em função de doses de zinco aplicadas $\left(\mathrm{mg} \mathrm{dm}^{-3}\right)$, em três fontes diferentes, nos solos LE e PV, em dois valores de $\mathrm{pH}, \mathrm{e}$ LV em apenas um valor de pH

\begin{tabular}{|c|c|c|c|}
\hline Solo & pH & E quação & $\mathbf{R}^{2}$ \\
\hline \multirow[t]{9}{*}{ LE } & & $\mathrm{ZnCl}_{2}$ & \\
\hline & 4,38 & $\hat{\mathrm{Y}}=-0,012400+0,003966 * * * \mathrm{X}$ & 0,939 \\
\hline & 5,40 & $\hat{\mathrm{Y}}=0,001400+0,000106^{* *} \mathrm{X}$ & 0,910 \\
\hline & & $\mathrm{ZnSO}_{4}$ & \\
\hline & 4,38 & $\hat{Y}=-0,001800+0,002617^{* * *} X$ & 0,987 \\
\hline & 5,40 & $\hat{\mathrm{Y}}=0,002200+0,000103 * \mathrm{X}$ & 0,926 \\
\hline & & ZnEDTA & \\
\hline & 4,38 & $\hat{Y}=-0,003400+0,002509 * * * X$ & 0,956 \\
\hline & 5,40 & $\hat{Y}=0,001400+0,000106 * * X$ & 0,910 \\
\hline \multirow{9}{*}{ PV } & & $\mathrm{ZnCl}_{2}$ & \\
\hline & 4,87 & $\hat{Y}=-0,000400+0,004237 * * * X$ & 0,995 \\
\hline & 6,00 & $\hat{Y}=0,001973+0,000321 * X+0,000009 * * X^{2}$ & 0,999 \\
\hline & & $\mathrm{ZnSO}_{4}$ & \\
\hline & 4,87 & $\hat{\mathrm{Y}}=-0,000600+0,003120 * * * \mathrm{X}$ & 0,996 \\
\hline & 6,00 & $\hat{\mathrm{Y}}=0,001600+0,000480 * * * \mathrm{X}$ & 0,998 \\
\hline & & ZnE DTA & \\
\hline & 4,87 & $\hat{Y}=-0,002000+0,003120 * * * X$ & 0,996 \\
\hline & 6,00 & $\hat{Y}=0,002164+0,000172 X+0,000007 * X^{2}$ & 0,998 \\
\hline \multirow[t]{6}{*}{ LV } & & $\mathrm{ZnCl}_{2}$ & \\
\hline & 4,64 & $\hat{Y}=0,003745-0,001417 X+0,000698^{* * *} X^{2}$ & 0,999 \\
\hline & & $\mathrm{ZnSO}_{4}$ & \\
\hline & 4,64 & $\hat{Y}=-0,009600+0,004734 * * * X$ & 0,993 \\
\hline & & ZnEDTA & \\
\hline & 4,64 & $\hat{Y}=0,003800+0,002183^{* * *} X$ & 0,993 \\
\hline
\end{tabular}

$*, * * \mathrm{e}^{* * *}$ significativos a 5,1 e $0,1 \%$, respectivamente.

maior estabilidade de mol éculas deZnEDTA. No solo de textura arenosa, foi observado maior fluxo difusivo de $\mathrm{ZnSO}_{4}$ em relação ao $\mathrm{ZnEDTA}$.

Nas amostras com aplicação de corretivo, ofluxo difusivo para as fontes $\mathrm{ZnCl}_{2}$, ZnEDTA e $\mathrm{ZnSO}_{4}$ obedeceu à ordem $P V>L E$, o que confirma o menor fluxo difusivo de $\mathrm{Zn}$ para solos com maior teor de argila.

O fluxo difusivo de $Z n$ em função do íon acompanhante na presença do corretivo obedeceu à seguinte ordem de grandeza para o solo $\mathrm{PV}: \mathrm{Cl}^{-}$ $>\mathrm{SO}_{4}{ }^{2-}>$ EDTA e para o solo LE: $\mathrm{SO}_{4}{ }^{2-}>$ EDTA $=$ $\mathrm{Cl}^{-}$. A maior movimentação de $Z n$ no LE na forma de $\mathrm{ZnSO}_{4}$ é explicada pela menor adsorção de $\mathrm{SO}_{4}{ }^{2-}$ no solo quando da el evação do pH, como sugere Dias (1992), tendo como conseqüência o efeito de íon acompanhante $\mathrm{SO}_{4}{ }^{2-}$.

O fluxo difusivo de $\mathrm{Zn}$ aumentou linearmente, na maioria dos casos, com as doses aplicadas em condições de pH mais baixo (Quadro 4), tendência freqüentemente observada na literatura (Elgawhary et al., 1970a,b; Ellis et al., 1970; Sharma \& Deb, 1988).

\section{CONCLUSÕES}

1. A variação do $\mathrm{pH}$ do solo demonstrou ser um fator de grande importância no controle do fluxo difusivo do zinco no solo, ocasionando grande diminuição de seus val ores com o aumento do pH.

2. Demodogeral, ofluxo difusivo de $\mathrm{Zn}$ foi menor com o aumento do teor de argila do solo.

3. O fluxo difusivo de Zn foi maior, nos três solos, quando a fonte utilizada foi $\mathrm{ZnCl}_{2}$.

\section{LITE RATURA CITADA}

ALVAREZ V., V.H. Caracterização química de solos. Viçosa, Universidade F ederal de Viçosa, 1985. 77p. 
ALVAREZ V., V.H. Efecto de los factores cantidad, intensidad y capacidad amortiguadora de fosfatos em la evaluación del fósforo disponible de suel os derivados de cenizas vol cánicas de la Meseta Tarasca. Chapingo: Col egio de Posgraduados, 1982. 300p.(Tese de Doutorado)

BEAR, F.E. Chemistry of soil. New York, 1967.p.85-106.

BRAGA, J .M. \& DEFELIPO, B.V. Determinação espectrofotomé trica de fósforo em extratos de solos e plantas. R. Ceres, 21:73-85, 1974.

CAVALLARO, N. \& McBRIDE, M.B. Zinc and copper sorption and fixation by an acid soil clay: effect of selective dissolutions. Soil Sci. Soc. Am. J ., 48:1050-1055, 1984.

CLARKE, A.L. \& GRAHAM, E.R. Zinc diffusion and distribution coefficients in soil as affected by soil texture, zinc concentration and pH. Soil Sci., 105: 409-418, 1968.

CUNHA, R.C.A. Retenção e movimento de zinco em solos do estado de São Paulo. Piracicaba, Escola Superior de Agricultura Luiz de Queiroz, 1989. 115p. (Tese de Mestrado)

DEFELIPO, B.V. \& RIBEIRO, A.C. Análise química de solo (metodologia). Viçosa, U niversidadeF ederal deViçosa, 1981. 17p. (Boletim de Extensão, 29)

DIAS, L.E. Dinâmica de formas de enxofre e de cátions trocáveis em col unas de sol o tratadas com diferentes doses de fósforo e de gesso. Viçosa, Universidade Federal de Viçosa, 1992. 147p. (Tese de Doutorado)

ELGABALY, M.M. Mechanism of zinc adsorption by colloidal clays and related minerals. Soil Sci., 69:167-173, 1950.

ELGAWHARY, S.M.; LINDSAY, W.L. \& KEMPER, W.D. Effect of complexing agents and acids on the diffusion of zinc to a simulated root. Soil Sci. Soc. Am. Proc., 34:211-214, 1970a.

ELGAWHARY, S.M.; LINDSAY, W.L. \& KEMPER, W.D. Effect of EDTA on the self-diffusion of zinc in aqueous solution and in soil. Soil Sci. Soc. Am. Proc., 34:66-70, 1970b.

ELLIS, B.G. \& KNEZEK, B.D. Adsorption reactions of micronutrients in soils. In: MORTVEDT, I.J .; GIORDANO, P.M. \& LINDSAY, W.L., eds. Micronutrients in Agriculture. Wisconsin, Soil Science Society of America, 1972. p.59-78.

ELLIS, J .H.; BARNHISEL, R.I \& P PHILLIPS, R.E. The diffusion of copper, manganese, and zinc as affected by concentration, clay mineralogy, and associated anions. Soil Sci. Soc. Am. Proc., 34:866-870, 1970.

EMPRESA BRASILEIRA DE PESQUISA AGROPECUÁRIA EMBRAPA. Serviço Nacional de Levantamento e Conservação de Solos. Manual de métodos de análise de solos. Rio de J aneiro, 1979. não paginado.

FERNANDES, B. \& SYKES, J .O. Capacidade decampo eretenção de água em três solos de Minas Gerais. R. Ceres, 15:1-39, 1968.

FERREIRA, M.E. \& CRUZ, M.C.P., eds. Micronutrientes na agricultura. Piracicaba, POTAFOS/CNPq, 1991. 734p. (Anais dol Simpósio sobre Micronutrientes na Agricultura).

KUMARI, K.; SINGH, R.P. \& SAXENA, S.K. Effect of different factors on the movement of copper and zinc in soils. J . Ind. Soc. Soil Sci., 36:346-348, 1988.

LIANG, J . \& KARAMANOS, R.E. DTPA-ExtractableFe, Mn, Cu, and Zn. In: CARTER, M.R., ed. Soil sampling and methods of analysis. Boca Raton, Lewis, 1993. p.87-90.
LINDSAY, W.L. Chemical equilibria in soils. New York, J ohn Wiley $\&$ Sons, 1979. 449p.

LINDSAY, W.L. I norganic equilibria affecting micronutrients in soils. In: MORTVEDT, J .J .; COX, F.R.; SHUMAN, L.M. \& WELCH, R.M., eds. Micronutrients in agriculture. Wisconsin, Soil Science Society of America, 1991. p.94-112.

LINDSAY, W.L. Zinc in soils and plant nutrition. Adv. Agron., 24:147-186, 1972.

MACHADO, P.L.O.A. \& PAVAN, M.A. Adsorção de zinco por alguns sol os do Paraná. R. Bras. Ci. Solo, 11:253-256, 1987.

MAGUIRE, M.; SLAVEK, I, VIMPANU, I.; HIGGINSON, R.R. \& PICKERING, W.F. Influence of $\mathrm{pH}$ on copper and zinc uptake by soil clays. Aust. J . Soil Res., 19:217-229, 1981.

MARSCHNER, H. Zinc uptake from soils. In: ROBSON, A.D., ed. In: INTERNATIONAL SYMPOSIUM ON ZINC IN SOILS AND PLANTS, 1993, Perth. Proceedings. Perth, 1993. p.59-71.

MEHLICH, A. Mehlich-3 soil test extractant: a modification of Mehlich-2 extractant. Comm. Soil Sci. Plant Anal., 15:14091416, 1984

MORAGHAN, J .T. \& MASCAGNI J r., H.J . Environimental and soil factors affeting micronutrient deficiencies and toxicities. In:MORTVEDT, J . .; COX, F.R.; SHUMAN, L.M. \& WELCH, R.M., eds. Micronutrients in agriculture. 2.ed. Wisconsin, Soil Science Society of America, 1991. p.371-425.

OLIVER, S. \& BARBER, S.A. Mechanisms for the movement of $\mathrm{Mn}, \mathrm{Fe}, \mathrm{B}, \mathrm{Cu}, \mathrm{Zn}, \mathrm{Al}$ and $\mathrm{Sr}$ from one soil to surface of soybean roots (Glycinemax) Soil Sci. Soc. Am. Proc., 30:468472,1966

RAIJ , B. \& QUAGGIO,J .A. Métodos de análise de sol os para fins de fertilidade. Campinas, Instituto Agronômico, 1983. 31p. (Boletim, 81)

SHARMA, K.N. \& DEB, D.L. Effect of soil moisture tension and soil compaction on self diffusion coefficient of zinc in soils of varying texture. J . Nucl. Agric. Biol., 13:118-120, 1984.

SHARMA, K.N. \& DEB, D.L. Calibration for zinc requirements of wheat based on diffusive flux equations. J . Nucl. Agric. Biol., 16:55-64, 1987.

SHARMA, K.N. \& DEB, D.L. Diffusion of zinc as affected by graded levels of zinc addition in soils of varying texture. J. Ind. Soc. Soil Sci., 36: 30-34, 1988.

SHARMA, K.N. \& DEB, D.L. Uptake of zinc by wheat plants in soils in relation to their diffusion coefficients under varying physical and chemical environment. II. Effect of soil bulk density. J . Nucl. Agric. Biol., 19:29-33, 1990.

SINGH, S.P.; SINHA, M.K. \& RANDHAWA, N.S. Diffusion of $65 \mathrm{Zn}$ as influenced by rates of applied zinc in soils of divergent texture. J . Ind. Soc. Soil Sci., 28:290-294, 1980.

TAM, K.H. Principles of soil chemistry. New York, Marcel Dekker, 1992. 362p.

TISDALE, S.L.; NELSON, W.L. \& BEATON, J .D. Soil fertility and fertilyzers. New York, Mcmillan, 1985. 754p.

WU, X.; AASEN, I. \& SELMER-OLSEN, A.R. A study of extraction methods for assessing soil zinc availability: I. Soil zinc extractability and soil zinc buffering capacity in relation to soil properties. Nor. J . Agric. Sci., 5:89-107, 1991. 